\title{
JS001, an anti-PD-1 mAb for advanced triple negative breast cancer patients after multi-line systemic therapy in a phase I trial
}

\author{
Li Bian ${ }^{1}$, Huiqiang Zhang ${ }^{1}$, Tao Wang ${ }^{1}$, Shaohua Zhang ${ }^{1}$, Haifeng Song ${ }^{2}$, Mingli Xu ${ }^{3}$, Sheng Yao ${ }^{4}$, \\ Zefei Jiang $^{1}$
}

${ }^{1}$ Department of Breast Oncology, The Fifth Medical Center of Chinese PLA General Hospital, Beijing 100071, China; ${ }^{2}$ National Engineering Research Center for Protein Drugs, Beijing Institute of Lifeomics, Beijing 102206, China; ${ }^{3}$ Beijing Halma Orient Medicine Technology Co., LTD, Beijing 100088, China; ${ }^{4}$ Shanghai Junshi Biosciences Co., LTD, Shanghai 201203, China

Contributions: (I) Conception and design: Z Jiang, H Song, S Yao; (II) Administrative support: Z Jiang, L Bian, M Xu; (III) Provision of study materials or patients: All authors; (IV) Collection and assembly of data: L Bian, H Zhang; (V) Data analysis and interpretation: Z Jiang, L Bian, H Zhang, S Yao; (VI) Manuscript writing: All authors; (VII) Final approval of manuscript: All authors.

Correspondence to: Zefei Jiang. Department of Breast Oncology, The Fifth Medical Center of Chinese PLA General Hospital, No.8 Dongda Street, Fengtai District, Beijing 100071, China. Email: jiangzefei@csco.org.cn.

Background: Triple negative breast cancer (TNBC), as defined by ER, PR and HER2 negative expression in tumor, has limited treatment options beyond conventional chemotherapy. JS001, a humanized IgG4 antibody for PD-1, has demonstrated acceptable safety profile and preliminary anti-tumor activity in solid tumors.

Methods: This phase I open-label study is designed to evaluate the safety, tolerability, and antitumor activity of JS001 in advanced TNBC patients who are refractory to standard systemic therapy. The study has a 3+3 dose escalation design with planned cohorts at 1,3, and $10 \mathrm{mg} / \mathrm{kg}$ Q2W followed by a dose expansion cohort at $3 \mathrm{mg} / \mathrm{kg}$. (Clinical Trial ID: NCT02838823).

Results: From August 04, 2016 to October 26, 2017, 20 heavily-pretreated advanced TNBC patients were enrolled into three dose cohorts ( 6 in $1 \mathrm{mg} / \mathrm{kg}, 8 \mathrm{in} 3 \mathrm{mg} / \mathrm{kg}$ and 6 in $10 \mathrm{mg} / \mathrm{kg}$ ). As of August 30, 2018, no DLT was observed and no MTD was reached. No AEs were grade 4 or 5 . The most common treatment related AEs were all grade 1/2. Treatment related grade 3 AEs (15\%) included 1 hyponatremia, 1 rash and 1 bronchospasm (infusion related reaction). Among 20 evaluable subjects, the ORR was $5 \%$. One patient in $10 \mathrm{mg} / \mathrm{kg}$ group obtained PR, who was PD-L1 strong positive (>50\%) in tumor biopsy, with treatment duration of 12.8 months as of data cutoff. As of follow-up on July 15, 2019, the patient continued PR with treatment duration of 24 months and still ongoing. Six patients achieved SD, for a DCR of $35 \%$. The median PFS of all subjects was 1.8 months (95\% CI, 1.4 to 4.6$) .45 \%$ subjects are PD-L1 positive $(\geq 1 \%$ cutoff), among whom a $11.1 \%$ ORR and a $22.2 \%$ DCR were observed.

Conclusions: JS001 exhibited a favorable safety profile in advanced TNBC patients who are refractory to multi-line systemic therapy. JS001 also showed a moderate response in these TNBC patients who had limited treatment options.

Keywords: JS001; anti-PD-1 mAb; triple negative; advanced breast cancer

Submitted Aug 12, 2019. Accepted for publication Aug 21, 2019.

doi: 10.21037/atm.2019.09.08

View this article at: http://dx.doi.org/10.21037/atm.2019.09.08 


\section{Introduction}

Triple negative breast cancer (TNBC) is defined by $\mathrm{ER}, \mathrm{PR}$ and HER2 negative in tumor. TNBC is about $15-20 \%$ in all types of breast cancer in Asian (1) and more common in younger women those with BRCA1/2 germline mutations (2). As lack of specific therapy targets, the main regimens for advanced TNBC are conventional chemotherapy (3). The patients with TNBC usually experienced the chemotherapy of anthracycline and taxane in adjuvant and/or neoadjuvant stage and platinum-based regimens as the preference in metastatic disease stage. It's lacking novel therapy drugs for these advanced TNBC patients who is refractory and/or intolerant to standard systemic therapy. The programmed death 1 (PD-1), an immune checkpoint receptor expressed on activated immunocyte, limits autoimmunity by regulating the activity of effector T cells (4-6). The anti-PD-1 antibody, which binds PD-1 and blocks the interaction between PD-1 and its ligands, has demonstrated antitumor activity in multiple tumor types including metastatic breast cancer, especially TNBC (7-11). In the phase 1b KEYNOTE-012 trial, the safety and anti-tumour activity of single-agent PD-1 inhibitor pembrolizumab given in advanced TNBC patients were evaluated. The result showed an acceptable safety profile and preliminary evidence of anti-tumour activity (12).

JS001 is the first monoclonal antibody (mAb) against programmed cell death protein-1 (PD-1) approved by the China Food and Drug Administration (CFDA) into the clinical trials. Pre-clinical pharmacological and pharmacokinetic (PK) data are encouraging and provide a basis for the JS001 in clinical trials (13). In 2017 ASCO Annual Meeting (Abstract 3067), a phase I clinical trial (NCT02836795) about the safety and efficacy of JS001 in advanced solid tumors was reported. JS001 exhibited a favorable safety and tolerability profile and promising antitumor activity, especially in previously under-evaluated acral and mucosal melanomas. In this study, we present the results from phase I study of single-agent JS001 for patients with advanced TNBC.

\section{Methods}

\section{Study design and population}

This study was an open-label, single centre, phase I, dose escalation study designed to evaluate the safety, tolerability, and antitumor activity of JS001 in patients with advanced TNBC (Clinical Trial ID: NCT02838823). The study has a $3+3$ dose escalation design with planned cohorts at 1,3 , and $10 \mathrm{mg} / \mathrm{kg}$ Q2W followed by a dose expansion cohort at $3 \mathrm{mg} / \mathrm{kg}$ Q2W. Key eligibility requirements included: age between 18 years and 70 years; estrogen receptornegative, progesterone receptor-negative, HER2-negative, recurrent or metastatic breast cancer which is refractory to standard systemic therapy; measurable disease according to Response Evaluation Criteria in Solid Tumors (RECIST) v1.1; Eastern Cooperative Oncology Group (ECOG) performance status of 0 to 1 ; patients were required to have adequate organ function; any number of prior systemic treatments. Key exclusion criteria included: prior therapy with immunologic checkpoint inhibitor; use of anti-tumor systemic therapy (including steroids and immunotherapy) within 4 weeks of study entry; active brain metastases (treated and stable brain metastases were allowed);active autoimmune disease. The study protocol and all amendments were approved by the ethics committees at the institution. All patients signed written informed consent. The study was conducted in accordance with the Good Clinical Practice guidelines.

\section{Treatment}

Sequential cohorts of at least three and no more than six patients received JS001 at 1,3 , or $10 \mathrm{mg} / \mathrm{kg}$ intravenously every 2 weeks. Dose-limiting toxicity (DLT) was defined as a treatment-related grade $\geq 3 \mathrm{AE}$ or laboratory abnormality occurring $\leq 28$ days postdose. The MTD was the highest dose at which no more than one of three or six patients experienced a DLT. Then, after dose escalation period, other no more than six patients were enrolled at a dose expansion at $3 \mathrm{mg} / \mathrm{kg}$ to confirm safety. Patients without DLT went on with JS001 therapy until unacceptable toxicity or disease progression.

\section{Assessments}

All patients were evaluated for toxicities based on Common Terminology Criteria for Adverse Events, version 4.0 (CTCAE4.0) during study treatment. All of Adverse Events (AE) and Serious AEs were collected for up to 90 days after the last JS001 dose. Antitumor activity of JS001 was assessed according to Response Evaluation Criteria in Solid Tumors, version 4.0 (RECIST 1.1) and immune-related response criteria (irRC) every 8 weeks. If clinically stable, patients with first imageology evidence of disease progression were permitted to continue on JS001 until a second scan 
performed 4 weeks later confirmed progression. Overall response rate (ORR), defined as percentage of patients with a best overall response of complete response (CR) or partial response (PR). Disease control rate (DCR), defined as the sum of CR, PR and stable disease (SD). Progression-free survival (PFS), defined as time from enrollment to disease progression or death.

PD-L1 expression was assessed in formalin-fixed, paraffin-embedded archival tumor samples at a central laboratory using a prototype immunohistochemistry assay and the antihuman PD-1 RM311 antibody (Cat No. 20171110, Suzhou Junmeng Bioscience Co., LTD, China). PD-L1 positivity was defined as PD-L1 expression in $\geq 1 \%$ tumor cells.

Circulating tumor cells (CTCs) was detected by the CellCollector device (Cat No. PR11072017-001, GILUPI $\mathrm{GmbH}$, Germany). It enables a directly in vivo isolation of CTCs from the vein of patients. The device is exposed to approximately $1 \mathrm{~L}$ of blood during half an hour, which technically increases the chance to capture CTCs. We used CellCollector to dynamically collect CTCs and planned to do CTC PD-L1 test.

Whole exome sequencing (WES) was performed with Agilent SureSelectXT Human All Exon V6 kit (Cat No. 5190-8864, Agilent Technologies Inc, USA) on FFPE tumor samples. In brief, sufficient amount of DNA was extracted from unstained FFPE sections with tumor content no less than $20 \%$, and was fragmented to $\sim 250$ bp by sonication. Library was constructed and hybridization capture was performed following manufacturer's instructions. Paired-end sequencing $(2 \times 150 \mathrm{bp})$ was performed on Illunima NovaSeq 6000. The genomic alterations including single base substitution, short and long indels, copy number variations, and gene rearrangement and fusions were assessed. The tumor mutation burden (TMB) was estimated by counting somatic mutations including coding base substitution and indels per megabase of the sequence examined. Driver gene mutations and known germline alterations in dbSNP were excluded from the TMB calculation.

\section{Statistical analysis}

The comparison of PFS between the two groups (PD-L1 $\geq 1 \%$ vs. PD-L1 <1\%) was analyzed using the log-rank test. Statistical test was considered significant with $\mathrm{P}<0.05$. The Kaplan-Meier method was applied for delineation of the PFS curve. Statistical analyses were conducted with available data using SAS version 9.4 (SAS
Institute Inc., Cary, NC).

\section{Results}

\section{Patient characteristics}

From August 04, 2016 to October 26, 2017, 20 heavilypretreated advanced TNBC patients were enrolled into three dose cohorts [6 in $1 \mathrm{mg} / \mathrm{kg}, 8$ in $3 \mathrm{mg} / \mathrm{kg}$ ( 3 in dose escalation and 5 in dose expansion) and 6 in $10 \mathrm{mg} / \mathrm{kg}$ ] with median age of 48.5 years (range, 33 to 60 years). $70 \%$ of patients have visceral metastases with poor prognosis. Median systemic therapy line for metastatic disease prior to JS001 is 2 (range 0 to 5), with $40 \%$ of patients having received at least three lines. All patients have been treated with taxanes and 18 patients (90\%) have prior platinumbased chemotherapy (Table 1). Nine patients (45\%) had $\mathrm{PD}-\mathrm{L} 1$ positive expression (PD-L1 $\geq 1 \%$ ) in tumor samples.

\section{Safety and tolerability}

In this study, no DLT was observed and no MTD was reached. As of August 30, 2018, no AEs were grade 4 or 5. The emergence of AEs is not dose related. The adverse events (AEs) occurred in at least two patients or $\geq$ grade 2 occurred in at least one patient were summarized in Table 2. The most common treatment related AEs were all grade $1 / 2$, including rash (40\%), bone pain and/or myalgia (30\%), pruritus (25\%), hypertriglyceridemia (25\%), hypothyroidism (25\%), anemia (25\%), hyperthyroidism (20\%), ALT increased (20\%), AST increased (20\%), flu like symptoms (20\%), decreased appetite (15\%), pneumonitis $(10 \%)$, thyroid-stimulating hormone (TSH) increased (10\%), hypertension (10\%), hyperglycemia (10\%), thyroid-stimulating hormone (TSH) decreased (10\%), hypocalcemia $(10 \%)$ and fatigue $(10 \%)$. Treatment related grade 3 AEs (15\%) included 1 hyponatremia, 1 rash and 1 bronchospasm (infusion related reaction). The patient developed acute bronchospasm at about 15 seconds of the first dose $(3 \mathrm{mg} / \mathrm{kg})$. Then JS001 infusion was stopped immediately. About 5 minutes after dexamethasone $5 \mathrm{mg}$ combination with doxofylline $300 \mathrm{mg}$ were administered, the symptoms of the patient disappeared. The patient received JS001 re-treatment 3 days later with pre-treatment of dexamethasone $5 \mathrm{mg}$ combination with diphenhydramine $20 \mathrm{mg}$ and didn't develop bronchospasm again. Three patients developed SAE. One case was the patient had grade 3 bronchospasm as before. One patient developed grade 2 
Table 1 Patient baseline characteristics

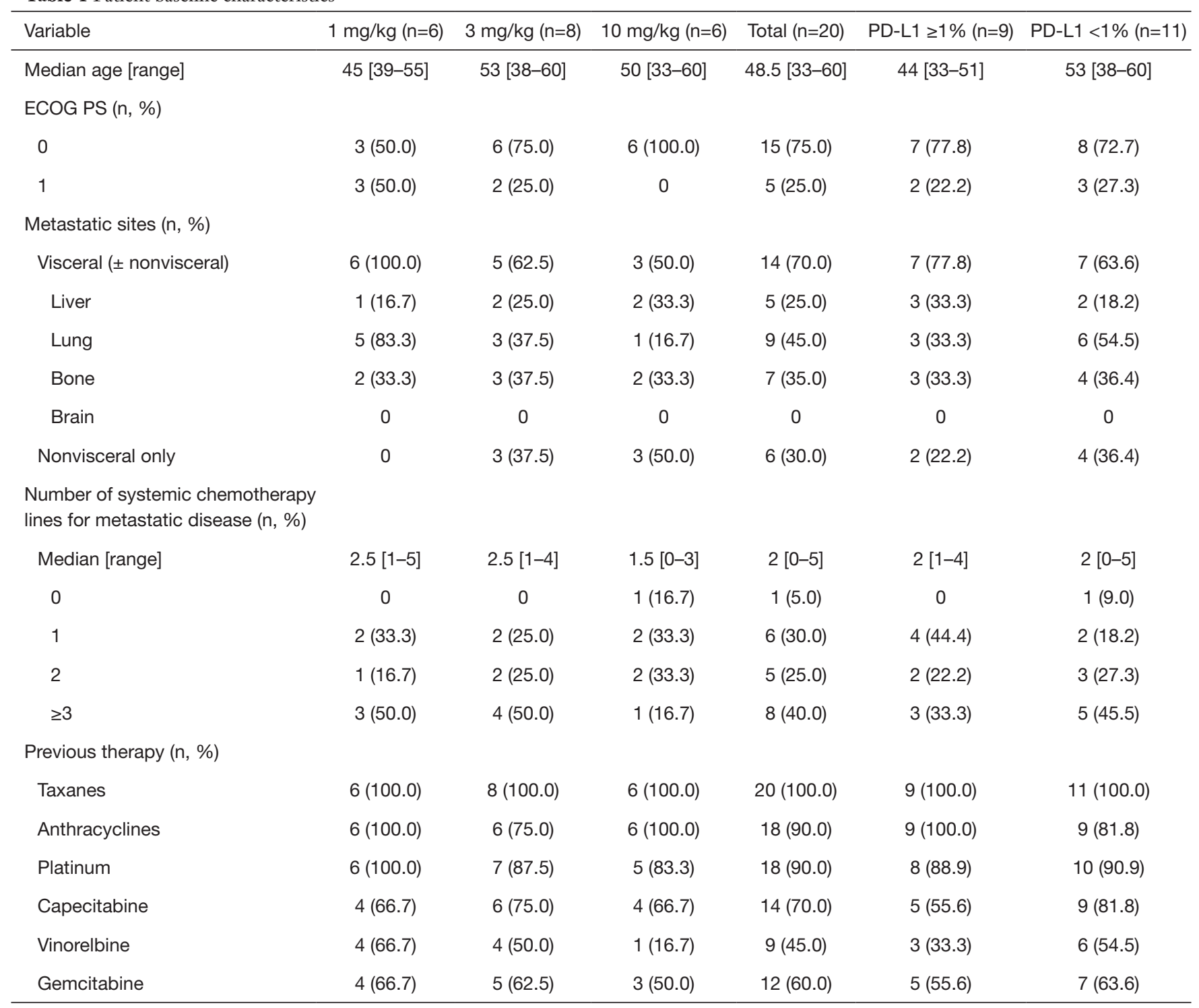

pneumonia following 7 doses $(3 \mathrm{mg} / \mathrm{kg})$ administered over 3 months. The patient was treated with JS001 interruption and steroids for 2 weeks, then pneumonia alleviated but not disappeared. The other patient experienced grade 3 rash, grade 2 ALT increased and AST increased, grade 2 serum adrenocorticotropic hormone (ACTH) decreased and cortisol decreased, grade 2 papilledema and hypophosphatemia, grade 1 hypothyroidism and pituitary involution in MRI following 4 doses $(10 \mathrm{mg} / \mathrm{kg})$ administered about 1.5 months. After JS001 withdrawal and hormone replacement, the levels of serum ACTH and cortisol of this patient were improved.

\section{Antitumor activity}

Among 20 evaluable subjects, the ORR was $5 \%$. One patient in $10 \mathrm{mg} / \mathrm{kg}$ group obtained PR with treatment duration of 55.9 weeks (12.8 months) as of data cutoff. As of follow-up on July 15, 2019, the patient continued PR with treatment duration of 24 months and still ongoing. Six patients achieved SD, for a DCR of $35 \%$ (Table 3). Among these 6 patients, 3 had SD over 6 months, one in $1 \mathrm{mg} / \mathrm{kg}$ group discontinued treatment for progression in lung lesions and the treatment duration of 35.7 weeks (8.2 months). The other two SD gainers (in $10 \mathrm{mg} / \mathrm{kg}$ group and $3 \mathrm{mg} / \mathrm{kg}$ group respectively) remained on study 
Table 2 Treatment-related adverse events that occurred in at least two patients or $\geq$ grade 2 occurred in at least one patient

\begin{tabular}{|c|c|c|c|c|c|c|c|c|}
\hline Variable & \multicolumn{2}{|c|}{$1 \mathrm{mg} / \mathrm{kg}(\mathrm{n}=6)$} & \multicolumn{2}{|c|}{$3 \mathrm{mg} / \mathrm{kg}(\mathrm{n}=8)$} & \multicolumn{2}{|c|}{$10 \mathrm{mg} / \mathrm{kg}(\mathrm{n}=6)$} & \multicolumn{2}{|c|}{ Total $(n=20)$} \\
\hline Rash & $2(33.3)$ & 0 & $2(25.0)$ & 0 & $4(66.7)$ & $1(16.7)$ & $8(40.0)$ & $1(5.0)$ \\
\hline Bone pain and/or Myalgia & $1(16.7)$ & 0 & $4(50.0)$ & 0 & $1(16.7)$ & 0 & $6(30.0)$ & 0 \\
\hline Pruritus & $2(33.3)$ & 0 & $1(12.5)$ & 0 & $2(33.3)$ & 0 & $5(25.0)$ & 0 \\
\hline Hypothyroidism & 0 & 0 & $3(37.5)$ & 0 & $2(33.3)$ & 0 & $5(25.0)$ & 0 \\
\hline Anemia & $1(16.7)$ & 0 & $3(37.5)$ & 0 & $1(16.7)$ & 0 & $5(25.0)$ & 0 \\
\hline Hyperthyroidism & $1(16.7)$ & 0 & $2(25.0)$ & 0 & $1(16.7)$ & 0 & $4(20.0)$ & 0 \\
\hline ALT increased & $1(16.7)$ & 0 & 0 & 0 & $3(50.0)$ & 0 & $4(20.0)$ & 0 \\
\hline Decreased appetite & 0 & 0 & $1(12.5)$ & 0 & $2(33.3)$ & 0 & $3(15.0)$ & 0 \\
\hline Pneumonitis & 0 & 0 & $1(12.5)$ & 0 & $1(16.7)$ & 0 & $2(10.0)$ & 0 \\
\hline $\begin{array}{l}\text { Thyroid-stimulating hormone } \\
\text { (TSH) increased }\end{array}$ & 0 & 0 & $1(12.5)$ & 0 & $1(16.7)$ & 0 & $2(10.0)$ & 0 \\
\hline Hypertension & 0 & 0 & $1(12.5)$ & 0 & $1(16.7)$ & 0 & $2(10.0)$ & 0 \\
\hline Hyperglycemia & 0 & 0 & $1(12.5)$ & 0 & $1(16.7)$ & 0 & $2(10.0)$ & 0 \\
\hline $\begin{array}{l}\text { Thyroid-stimulating hormone } \\
\text { (TSH) decreased }\end{array}$ & $1(16.7)$ & 0 & $1(12.5)$ & 0 & 0 & 0 & $2(10.0)$ & 0 \\
\hline Cortisol decreased & 0 & 0 & 0 & 0 & $1(16.7)$ & 0 & $1(5.0)$ & 0 \\
\hline Hypophosphatemia & 0 & 0 & 0 & 0 & $1(16.7)$ & 0 & $1(5.0)$ & 0 \\
\hline Papilledema & 0 & 0 & 0 & 0 & $1(16.7)$ & 0 & $1(5.0)$ & 0 \\
\hline Cholesterol high & 0 & 0 & $1(12.5)$ & 0 & 0 & 0 & $1(5.0)$ & 0 \\
\hline White blood cell decreased & 0 & 0 & 0 & 0 & $1(16.7)$ & 0 & $1(5.0)$ & 0 \\
\hline Proteinuria & 0 & 0 & $1(12.5)$ & 0 & 0 & 0 & $1(5.0)$ & 0 \\
\hline
\end{tabular}


Table 3 Efficacy in patients with advanced TNBC treated with JS001

\begin{tabular}{|c|c|c|c|c|c|c|}
\hline Variable & $1 \mathrm{mg} / \mathrm{kg}(\mathrm{n}=6)$ & $3 \mathrm{mg} / \mathrm{kg}(\mathrm{n}=8)$ & $10 \mathrm{mg} / \mathrm{kg}(\mathrm{n}=6)$ & Total $(n=20)$ & PD-L1 $\geq 1 \%(n=9)$ & PD-L1 $<1 \%(n=11)$ \\
\hline PR & 0 & 0 & $1(16.7)$ & $1(5.0)$ & $1(11.1)$ & 0 \\
\hline SD & $2(33.3)$ & $2(25.0)$ & 2 (33.3) & $6(30.0)$ & $1(11.1)$ & $5(45.5)$ \\
\hline PD & $4(66.7)$ & $6(75.0)$ & $3(50.0)$ & $13(65.0)$ & 7 (77.8) & $6(54.5)$ \\
\hline DCR (\%) (95\% Cl) & 33.3 (4.3 to 77.7$)$ & 25.0 (3.2 to 65.1$)$ & 50.0 (11.8 to 88.2$)$ & 35.0 (15.4 to 59.2$)$ & 22.2 (2.8 to 60.0$)$ & 45.5 (16.7 to 76.6$)$ \\
\hline $\begin{array}{l}\text { Median PFS (95\% } \\
\mathrm{Cl}, \text { months) }\end{array}$ & 2.1 (0.9 to 5.5 ) & 1.7 (1.0 to NE) & 2.7 (1.8 to NE) & 1.8 (1.4 to 4.6$)$ & 1.8 (1.0 to 3.7$)$ & 2.9 (1.0 to NE) \\
\hline $\begin{array}{l}\text { 6-month PFS } \\
\text { rate }(\%)(95 \% \mathrm{Cl})\end{array}$ & 0.0 (NE to NE) & 25.0 (3.7 to 55.8) & 33.3 (4.6 to 67.6$)$ & 18.0 (4.8 to 38.0 ) & 11.1 (0.6 to 38.8) & 22.7 (3.8 to 51.1$)$ \\
\hline
\end{tabular}

*, group PD-L1 $\geq 1 \%$ vs. group PD-L1 <1\%, P=0.327, HR =1.64 (0.6 to 4.46).

with treatment durations of 63.9 weeks ( 14.7 months) and 55.9 weeks (12.8 months) respectively as of data cutoff (Figure 1). The median PFS of all subjects was 1.8 months (95\% CI, 1.4 to 4.6), with a 6-month PFS rate of $18 \%$ (95\% CI, $4.8 \%$ to $38 \%$ ) (Figure $2 A$ ). $45 \%$ subjects are PD-L1 positive ( $\geq 1 \%$ cutoff), among whom a $11.1 \%$ ORR and a $22.2 \%$ DCR were observed. In PD-L1 positive subgroup, the median PFS was 1.8 months (95\% CI, 1.0 to 3.7), and meanwhile the median PFS was 2.9 months (95\% CI, 1.0 to $\mathrm{NE})$ in PD-L1 negative subgroup $[\mathrm{P}=0.327, \mathrm{HR}=1.64(0.6$ to 4.46)] (Figure 2B).

The patient who experienced a PR had previously received anthracycline and taxane as the neoadjuvant/ adjuvant therapy and docetaxel plus capecitabine follow by single capecitabine for metastatic disease. She had progress disease after the third cycle of single capecitabine and then she received JS001 $(10 \mathrm{mg} / \mathrm{kg})$ treatment every 2 weeks from July 12, 2017. After 24 weeks, CT evaluation revealed a remarkable reduction of the pleural metastasis that reached PR. After 32 weeks CT showed a further reduction of the disease that confirmed PR (Figure $3 A$ ). She was PD-L1 strong positive ( $>50 \%)$ in tumor biopsy (Figure 3B). We used CellCollector to dynamically collect CTCs from the patient (planned to do CTC PD-L1 test) on July 12, 2017 and November 15, 2017, unexpectedly we captured no CTCs from the patient. Tumor genomic profiling by Agilent whole exome sequencing revealed no significant single nucleotide variant (SNV) were found, meanwhile, some significant copy number variants $(\mathrm{CNV})$ were detected. FBXW7 gene deletion, CCND2 gene amplification, FGF23 gene amplification and FGF6 gene amplification were identified and predominantly associated with poor prognosis. The tumor sample from this patient had a tumor mutation burden (TMB) at $4.7 \mathrm{mut} / \mathrm{Mb}$ (Figure 3C).

\section{Discussion}

Triple negative breast cancer (TNBC) is a heterogeneous disease. Different subgroups of TNBC have been identified on the basis of genomic characteristics. Accordingly, new targeted therapeutic strategies are developed $(14,15)$. The proportion of TNBC patients with PD-L1 expression positive is the highest among all types of breast cancer. TNBC patients have high concentrations of tumourinfiltrating lymphocytes, which can induce the tumorspecific immune response (16). Furthermore, TNBC has the highest frequency of neoplastic mutations, which might increase the chance of immunogenic mutations generating neoantigens (17). All of these are the reasons why TNBC is regarded as the optimal subtype of breast cancers for immune checkpoint inhibition therapy. This phase I study is evaluating the safety and efficacy of the immune checkpoint inhibitor (anti-PD-1 mAb) JS001 in advanced TNBC patients who are refractory to multi-line conventional therapy.

Immune-related adverse events (irAEs) results from blockade of these immune checkpoints. Common toxicities involve the endocrine organs, lungs, liver and skin (18-20). In KEYNOTE-012, the incidence of grade 3 to 5 treatment-related AEs from single-agent pembrolizumab in advanced TNBC was $15.6 \%$ (12). In this study, we 


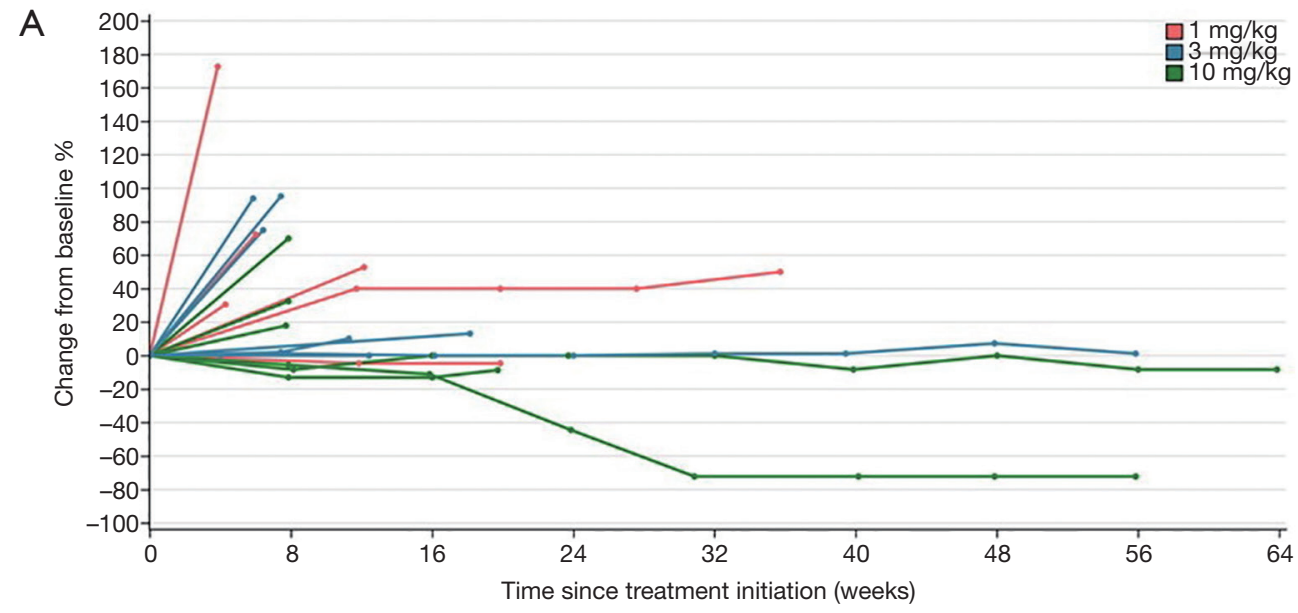

B

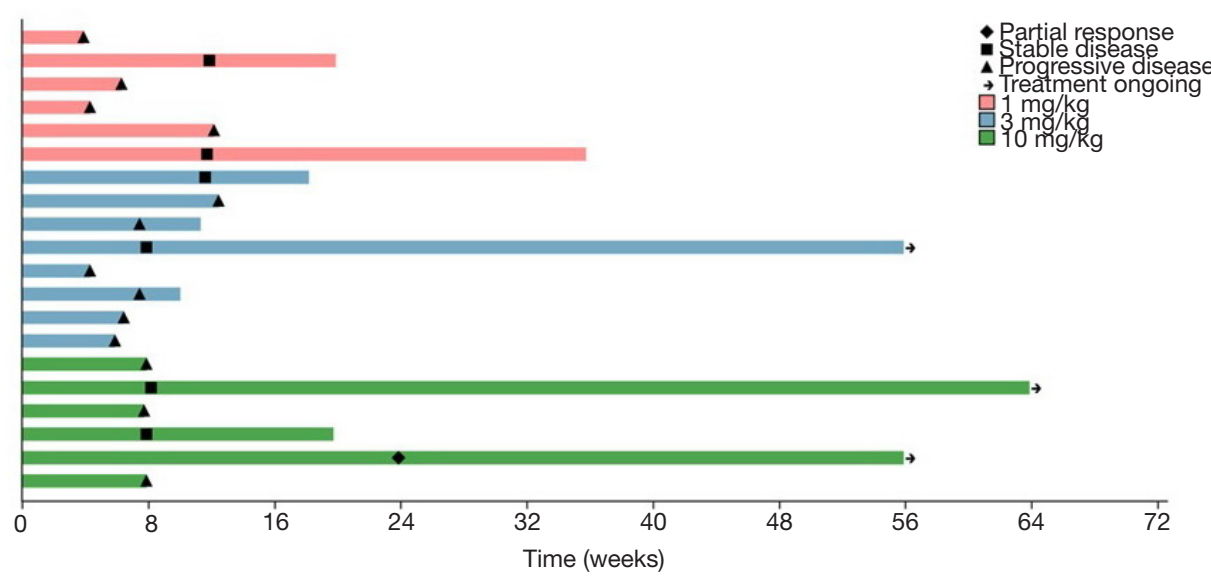

Figure 1 Tumour response to JS001 (n=20). (A) Change from baseline of target lesions over time. (B) The time on treatment for each patient and duration of response. Length of bars equals time from treatment initiation to last imaging assessment.

found that JS001 demonstrated an acceptable safety and tolerability profile. No DLT was observed and no MTD was reached. No AEs were grade 4 or 5 . The emergence of AEs is not dose related. The most common treatment related AEs were all grade 1/2. Treatment related grade 3 AEs included 1 hyponatremia, 1 rash and 1 bronchospasm (infusion related reaction) (15\%). All of the irAEs relieved after JS001 interruption and steroids treatment. In 2018 ESMO conference, the management of toxicities from immunotherapy: ESMO Clinical Practice Guidelines for diagnosis, treatment and follow-up was issued, which indicated immunotherapy should be discontinued immediately, and immunosuppressive or immune modulating drugs initiated promptly when the patient developed irAEs (21).

The response of PD-1 inhibitor single-agent in breast cancer is inferior to that in melanoma and lung cancer. Pembrolizumab demonstrated an ORR of $18.5 \%$ in KEYNOTE-012 study for advanced TNBC (12). In KEYNOTE-086 Cohort A study, ORR was $4.7 \%$, DCR was $7.6 \%$ and median PFS was 2 months for pembrolizumab (22). In this study about JS001, among 20 evaluable subjects, the ORR was $5 \%$. One patients in $10 \mathrm{mg} / \mathrm{kg}$ group obtained PR, who was PD-L1 strong positive $(>50 \%)$ in tumor biopsy, with treatment duration of 12.8 months as of data cutoff. As of follow-up on July 15, 2019, the patient continued PR with treatment duration of 24 months and still ongoing. 6 patients achieved SD, for a DCR of $35 \%$. Among these 6 patients, 3 had SD over 6 months, with the longest at 14.7 months as of data cutoff and still ongoing. In our study, the ORR of JS001 is lower than pembrolizumab in KEYNOTE-012. We analyzed the potential correlative factors including two points. First, all 20 enrolled patients are heavily pretreated. The median 

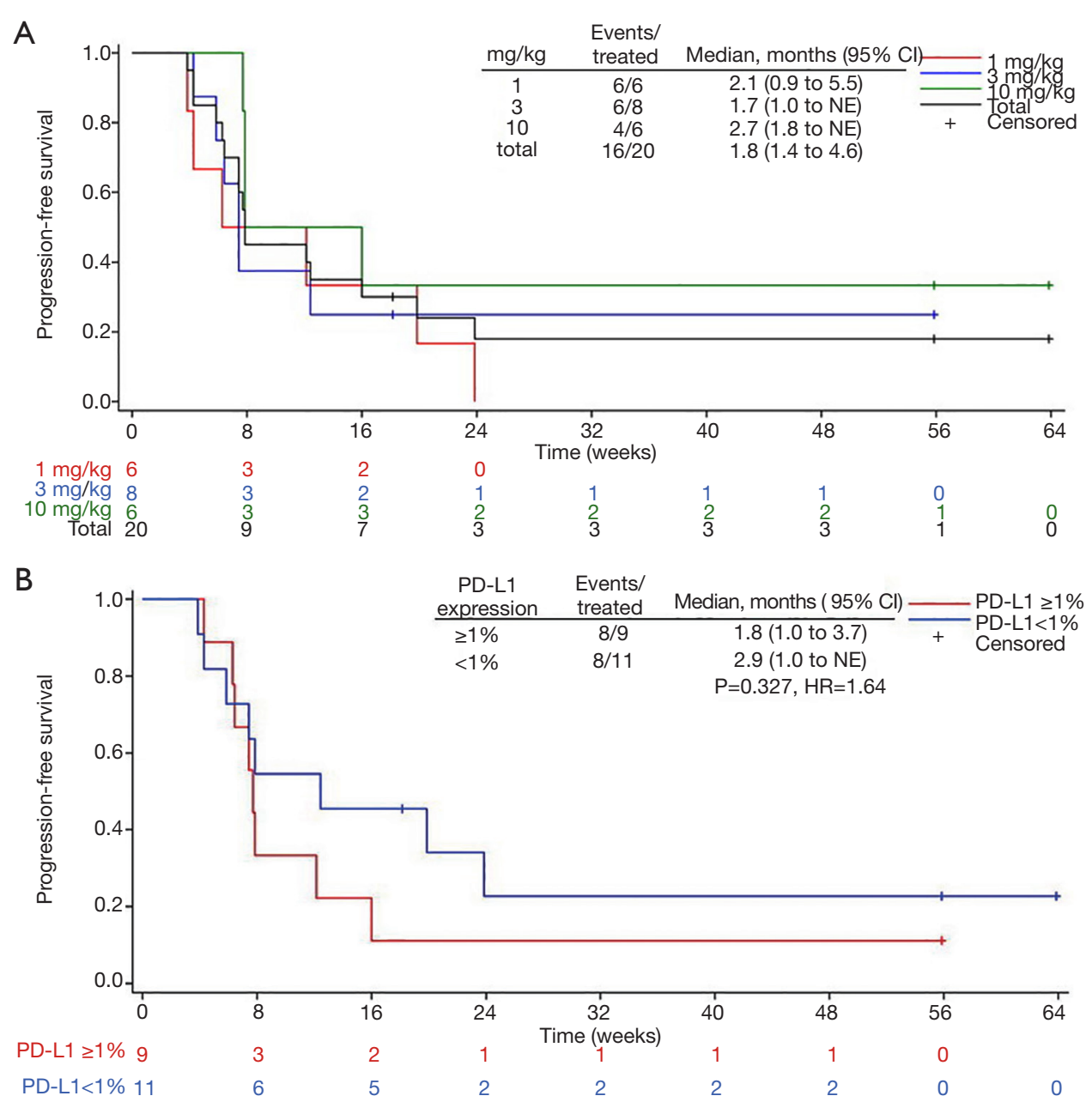

Figure 2 Kaplan-Meier curves of progression-free survival (PFS) in JS001 treated patients with advanced TNBC. (A) Analysis of three dose cohorts and total patients. (B) Analysis of PD-L1 positive vs. negative expression patients.

systemic line of the chemotherapy for advanced disease prior to JS001 is 2 and the most line is 5. All patients had previously received a taxane, $90 \%$ had also been exposed to platinum and anthracyclines, and the majority had also been exposed to vinorelbine, capecitabine and gemcitabine. Second, 70\% enrolled patients have visceral disease with poor prognosis. Without cytotoxic therapy for these patients may be infeasible, because the disease frequently progress while waiting for immune checkpoint blockade to take effect. Nevertheless, we also see three patients have long time of disease control, which suggest JS001 single therapy is effective in suitable patient subgroup.

In our study, $45 \%$ subjects are PD-L1 positive $(\geq 1 \%$ cutoff), among whom a $11.1 \%$ ORR and a $22.2 \%$ DCR were observed. The median PFS was 1.8 months in PD-L1 positive subgroup and 2.9 months in $\mathrm{PD}-\mathrm{L} 1$ negative subgroup $(\mathrm{P}=0.327, \mathrm{HR}=1.64)$. However, the patient obtained PR is the only one with PD-L1 strong positive $(>50 \%)$ in tumor biopsy. Does this suggest we can identify the response patients subgroup of JS001 through PD-L1 high expression? In a phase 3 trial CheckMate 057 (23), patients with non-squamous NSCLC after failure of firstline platinum doublet chemotherapy received nivolumab versus docetaxel. A test for interaction suggested a strong predictive association between PD-L1 expression and clinical outcome at all expression levels for all efficacy end points. Among patients whose tumors expressed PD-L1 (at the $\geq 1 \%, \geq 5 \%$, and $\geq 10 \%$ expression levels), nivolumab nearly doubled median overall survival as compared with docetaxel. In a phase 3 trial KEYNOTE-024 (24), 305 patients who had previously untreated advanced non-small cell lung cancer (NSCLC) with PD-L1 $\geq 50 \%$ expression 

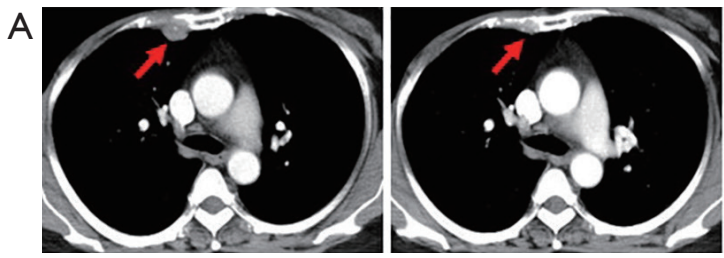

B
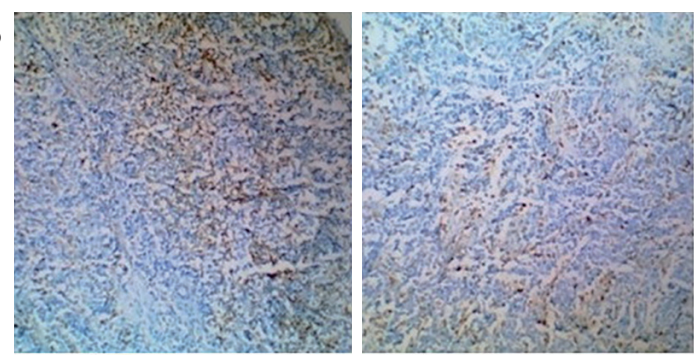
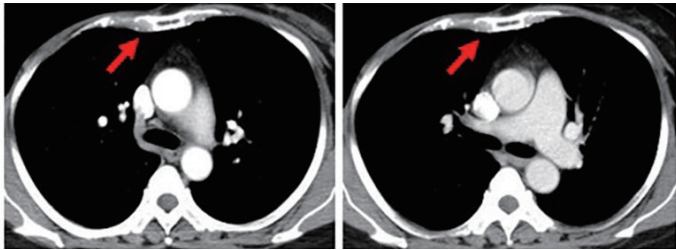

\begin{tabular}{|c|c|c|}
\hline Cene & Alternation type & Copy number \\
\hline FBXW7 & Gene deletion & 0 \\
\hline CCND2 & Gene amplification & 6 \\
\hline FGF23 & Gene amplification & 5 \\
\hline FGF6 & Gene amplification & 6 \\
\hline \multicolumn{3}{|c|}{ TMB 4.7 mut/Mb } \\
\hline
\end{tabular}

Figure 3 The advanced TNBC patient obtained partial response (PR) who received JS001 at a dose of $10 \mathrm{mg} / \mathrm{kg}$, with treatment duration of 55.9 weeks (12.8 months) as of data cutoff. As of follow-up on July 15, 2019, the patient continued PR with treatment duration of 24 months and still ongoing. (A) CT scan show the pleural metastasis (red arrow) at pretreatment, 24 weeks (PR), 32 weeks (PR confirmation) and 56 weeks (last imaging assessment) respectively. (B) PD-L1 strong positive ( $>50 \%)$ in tumor biopsy (10×) using a prototype immunohistochemistry assay. (C) Whole exome sequencing (WES) revealed some significant copy number variants (CNV) were detected and tumor mutation burden (TMB) at $4.7 \mathrm{mut} / \mathrm{Mb}$.

received either pembrolizumab or the platinum-based chemotherapy. Median PFS was 10.3 versus 6.0 months ( $\mathrm{HR}=0.50 ; \mathrm{P}<0.001)$. The estimated rate of overall survival at 6 months was $80.2 \%$ versus $72.4 \%(\mathrm{HR}=0.60 ; \mathrm{P}=0.005)$. However, further studies are needed to explore the correlation between PD-L1 high expression (for example $\geq 50 \%$ even higher) and the response to $\mathrm{PD}-1$ inhibitors in TNBC.

\section{Conclusions}

In summary, JS001 exhibited a favorable safety profile in advanced TNBC patients who are refractory to multi-line conventional therapy. JS001 also showed a moderate response in these TNBC patients who had limited treatment options, partial patients had disease control for a long time irrespective of PD-L1 status. Combination of JS001 with chemotherapy or anti-angiogenesis therapy are being evaluated for future clinical development in advanced TNBC patients.

\section{Acknowledgments}

Our sincere gratitude to all patients for their permission to use the data and pictures for this paper. This work was funded by Shanghai Junshi Biosciences Co., LTD.

\section{Footnote}

Conflicts of Interest: The authors have no conflicts of interest to declare.

Etbical Statement: This study was approved by the Ethics Committee of the medical center (June 28, 2016) and all patients signed informed consent form before screening. The authors are accountable for all aspects of the work (including full data access, integrity of the data and the accuracy of the data analysis) in ensuring that questions related to the accuracy or integrity of any part of the work are appropriately investigated and resolved.

\section{References}

1. Foulkes WD, Smith IE, Reis-Filho JS. Triple-negative breast cancer. N Engl J Med 2010;363:1938-48.

2. Peshkin BN, Alabek ML, Isaacs C. BRCA1/2 mutations and triple negative breast cancers. Breast Dis 2010;32:25-33.

3. Zhang $\mathrm{H}$, Jiang $\mathrm{Z}$. The role and optimization of chemotherapy for breast cancer in the era of classified therapy. Zhonghua Yi Xue Za Zhi 2014;94:2001-3.

4. Topalian SL. Targeting immune checkpoints in cancer 
therapy. JAMA 2017;318:1647-8.

5. Pardoll DM. The blockade of immune checkpoints in cancer immunotherapy. Nat Rev Cancer 2012;12:252-64.

6. Topalian SL, Drake CG, Pardoll DM. Targeting the PD-1/B7-H1(PD-L1) pathway to activate anti-tumor immunity. Curr Opin Immunol 2012;24:207-12.

7. Robert C, Schachter J, Long GV, et al. Pembrolizumab versus Ipilimumab in Advanced Melanoma. N Engl J Med 2015;372:2521-32.

8. Garon EB, Rizvi NA, Hui R, et al. Pembrolizumab for the treatment of non-small-cell lung cancer. N Engl J Med 2015;372:2018-28.

9. Muro K, Chung HC, Shankaran V, et al. Pembrolizumab for patients with PD-L1-positive advanced gastric cancer (KEYNOTE-012): a multicentre, open-label, phase 1b trial. Lancet Oncol 2016;17:717-26.

10. Seiwert TY, Burtness B, Mehra R, et al. Safety and clinical activity of pembrolizumab for treatment of recurrent or metastatic squamous cell carcinoma of the head and neck (KEYNOTE-012): an open-label, multicentre, phase 1b trial. Lancet Oncol 2016;17:956-65.

11. Plimack ER, Bellmunt J, Gupta S, et al. Safety and activity of pembrolizumab in patients with locally advanced or metastatic urothelial cancer (KEYNOTE-012): a nonrandomised, open-label, phase $1 \mathrm{~b}$ study. Lancet Oncol 2017;18:212-20.

12. Nanda R, Chow LQ, Dees EC, et al. Pembrolizumab in Patients With Advanced Triple-Negative Breast Cancer: Phase Ib KEYNOTE-012 Study. J Clin Oncol 2016;34:2460-7.

13. Fu J, Wang F, Dong LH, et al. Preclinical evaluation of the efficacy, pharmacokinetics and immunogenicity of JS-001, a programmed cell death protein-1 (PD-1) monoclonal antibody. Acta Pharmacol Sin 2017;38:710-8.

14. Denkert C, Liedtke C, Tutt A, et al. Molecular alterations in triple-negative breast cancer-the road to new treatment strategies. Lancet 2017;389:2430-42.

Cite this article as: Bian L, Zhang H, Wang T, Zhang S, Song H, Xu M, Yao S, Jiang Z. JS001, an anti-PD-1 mAb for advanced triple negative breast cancer patients after multiline systemic therapy in a phase I trial. Ann Transl Med 2019;7(18):435. doi: 10.21037/atm.2019.09.08
15. Shao F, Sun H, Deng CX. Potential therapeutic targets of triple-negative breast cancer based on its intrinsic subtype. Oncotarget 2017;8:73329-44.

16. Foulkes WD, Stefansson IM, Chappuis PO, et al. Germline BRCA1 mutations and a basal epithelial phenotype in breast cancer. J Natl Cancer Inst 2003;95:1482-5.

17. Budczies J, Bockmayr M, Denkert C, et al. Classical pathology and mutational load of breast cancer - integration of two worlds. J Pathol Clin Res 2015;1:225-38.

18. Naidoo J, Page DB, Li BT, et al. Toxicities of the antiPD-1 and anti-PD-L1 immune checkpoint antibodies. Ann Oncol 2015;26:2375-91.

19. Khoja L, Day D, Wei-Wu Chen T, et al. Tumour- and class-specific patterns of immune-related adverse events of immune checkpoint inhibitors: a systematic review. Ann Oncol 2017;28:2377-85.

20. Kumar V, Chaudhary N, Garg M, et al. Current diagnosis and management of immune related adverse events (irAEs) induced by immune checkpoint inhibitor therapy. Front Pharmacol 2017;8:49.

21. Haanen JBAG, Carbonnel F, Robert C, et al. ESMO Guidelines Committee. Management of toxicities from immunotherapy: ESMO Clinical Practice Guidelines for diagnosis, treatment and follow-up. Ann Oncol 2018;29: iv264-6.

22. Adams S. Phase 2 study of pembrolizumab (pembro) monotherapy for previously treated metastatic triple-negative breast cancer (mTNBC): KEYNOTE-086 cohort A. Paper presented at: ASCO 2017(Abstract1008); Chicago, USA.

23. Borghaei H, Paz-Ares L, Horn L, et al. Nivolumab versus Docetaxel in Advanced Nonsquamous Non-Small-Cell Lung Cancer. N Engl J Med 2015;373:1627-39.

24. Reck M, Rodríguez-Abreu D, Robinson AG, et al. Pembrolizumab versus Chemotherapy for PD-L1Positive Non-Small-Cell Lung Cancer. N Engl J Med 2016;375:1823-33. 